\title{
Hipercolesterolemia: un efecto metabólico después del bypass gástrico que aún falta por definir
}

\author{
Hypercholesterolemia: a metabolic effect after the gastric bypass that still needs to be \\ defined
}

\author{
Jesús Morales-Maza* y Jorge H. Rodríguez-Quintero \\ Servicio de Cirugía, Instituto Nacional de Ciencias Médicas y Nutrición Salvador Zubirán, Universidad Nacional Autónoma de México, Ciudad de \\ México, México
}

\begin{abstract}
A los editores:
Hemos leído con gran interés el trabajo publicado por el Dr. José D. Hernández-Marín, et al. ${ }^{1}$ en el número 4 del volumen 86 de 2018 de esta revista, que reporta los efectos metabólicos en pacientes obesos operados de bypass gástrico en su centro.

Queremos felicitar a los autores por los buenos resultados que han logrado en estos 5 años de experiencia.
\end{abstract}

Efectivamente, el bypass gástrico logra muy buenos resultados metabólicos; sin embargo, algunos efectos a largo plazo parecen no haberse definido con exactitud a la fecha actual, específicamente los valores de colesterol total (CT) y de lipoproteínas de baja densidad (LDL). Sobre este aspecto quisiéramos agregar un par de comentarios. Este efecto metabólico es un punto de debate ya que no se ha podido determinar si los pacientes intervenidos quirúrgicamente deben o no continuar consumiendo estatinas por el solo hecho de haber sido operados; actualmente esto se determina de acuerdo con los valores de LDL, y por eso se vuelve muy relevante analizar este efecto metabólico.

Los autores lograron observar a 12 meses de seguimiento los valores de CT y LDL en ambos grupos estudiados, pacientes con y sin diabetes tipo 2, y reportan una reducción que fue estadísticamente significativa. Estos resultados van de acuerdo con algunos estudios publicados.

Uno de los primeros metaanálisis que evaluó el efecto a largo plazo de la cirugía bariátrica reportó resultados favorables en el perfil lipídico en el subgrupo de pacientes tratados con bypass gástrico, con una mejoría de la dislipidemia a 10 años de seguimiento en más del $90 \%$ de los pacientes ${ }^{2}$.

En un estudio publicado en 2011 que estudió el efecto del bypass gástrico en 215 pacientes, con un seguimiento de hasta 42 meses, se reportó una disminución del CT, e incluso al comparar el bypass gástrico con la banda gástrica ajustable fue superior, logrando ser estadísticamente significativo ${ }^{3}$, a pesar de haber sido un estudio retrospectivo y que no fue diseñado para explorar de manera específica este efecto metabólico, fue un hallazgo relevante.

Sin embargo, a pesar de existir una percepción generalizada de buenos resultados en cuanto al control lipídico con la cirugía bariátrica, algunos estudios no han coincidido con estos resultados.

Un estudio que evaluó los efectos del bypass gástrico a 1 año de seguimiento no logró significancia estadística en la disminución de los valores de LDL ${ }^{4}$.

En otro estudio, publicado en 2004, con uno de los seguimientos más largos reportados, se compararon los resultados del bypass gástrico con el manejo médico y cambios de estilo de vida, y el número de pacientes con hipercolesterolemia fue muy similar en ambos grupos a los 2 años $(n=504)$ e incluso a los 10 años de seguimiento $(n=135)^{5}$.

Es por eso que, como los mismos autores del trabajo reconocen, sí vale la pena continuar realizando una evaluación de los efectos metabólicos a mayor plazo, ya que esto determinará si algunos parámetros

\footnotetext{
Correspondencia:

*Jesús Morales-Maza

Vasco de Quiroga, 15

Col. Sección XVI, Del. Tlalpan

C.P. 14080 , Ciudad de México, México

E-mail: jesus.moralesm1@ hotmail.com

Fecha de recepción: 20-08-2018

Fecha de aceptación: 24-08-2018

DOI: 10.24875/CIRU.18000673
}

Cir Cir. 2018;86:593-594

Contents available at PubMed www.cirugiaycirujanos.com 
remiten con la estrategia quirúrgica o permanecen alterados.

Por último, nuevamente quisiéramos felicitar a los autores de esta publicación por sus resultados tan favorables en sus pacientes tratados con bypass gástrico.

\section{Conflicto de intereses}

Los autores declaran no tener conflictos de intereses.

\section{Bibliografía}

1. Hernández-Marín JD, Marrufo-Patrón CA, López-Rosales F. Efectos metabólicos en pacientes obesos posoperados de bypass gástrico laparoscópico: 5 años de experiencia en un hospital de tercer nivel. Cir Cir. 2018;86:338-46.

2. Buchwald H, Avidor $\mathrm{Y}$, Braunwald E, Jensen MD, Pories W, Fahrbach $\mathrm{K}$, et al. Bariatric surgery: a systematic review and meta-analysis. JAMA. 2004;292:1724-37.

3. Pohle-Krauza RJ, McCarroll ML, Pasini DD, Dan AG, Zografakis JG. Age and gender exert differential effects on blood lipids in patients after LAGB and LRYGB. Surg Obes Relat Dis. 2011;7:170-5.

4. Raffaelli M, Guidone C, Callari C, laconelli A, Bellantone R, Mingrone G Effect of gastric bypass versus diet on cardiovascular risk factors. Ann Surg. 2014;259:694-9.

5. Sjöström L, Lindroos AK, Peltonen M, Torgerson J, Bouchard C, Carlsson B, et al. Lifestyle, diabetes, and cardiovascular risk factors 10 years after bariatric surgery. N Engl J Med. 2004;351:2683-93. 